\title{
Implementing a Recurrence Relation Model for Finding the General Form of a Special Integer Sequence Generated by Geometric Sequences with $t$ Representing the First Term and $p$ Representing the Ratio
}

\author{
$1^{\text {st }}$ Gatot Muhsetyo \\ Universitas Negeri Malang \\ Malang, Indonesia \\ gatot.muhsetyo.fmipa@um.ac.id
}

\begin{abstract}
The general form of an geometric sequence is $t, t p, t p^{2}, t p^{3}, \ldots, t p^{n-1}, \ldots$ This sequence is one of integer sequences. The rule for determining the nth term is $u_{n}=t p^{n-1}$. The specific sequence that is generated by this gemetric sequence is $t, t, t, \ldots$ It times], (tp), (tp), (tp),... I(tp) times], $\left(\mathrm{tp}^{2}\right),\left(\mathrm{tp}^{2}\right),\left(\mathrm{tp}^{2}\right), \ldots$ $\left[\left(t p^{2}\right)\right.$ times, ..., $\left(t p^{n-1}\right),\left(t p^{n-1}\right),\left(t p^{n-1}\right), \ldots\left[\left(t p^{n-1}\right)\right.$ times $] .$. .Theproblem is finding the rule for drciding its nth term. The purpose of this study is implementing a recurrence relation model for solving the problem. By the help of a linear recurrence relation with constant coefficients of $c_{o} u_{n}+c_{1} u_{n-1}+\ldots+c_{k} a_{n-k}=f(n)$, it can be found that $u_{n}=\left(1-\frac{t}{p-1}\right)+\left(\frac{t}{p^{2}-p}\right) \cdot p^{n}$
\end{abstract}

Keywords---generated, geometric, recurrence, sequence

\section{INTRODUCTION}

It is a fact that we frequently talk about a list integers as a representation of a set. The set has a functional connection with the set of natural numbers, and it is commonly known as integers sequences. An integer sequence is an ordered list of the integers. The notation of an integer sequence is written as a list, and separated by coma. As functional connections, or relations, each integer is regarded as the term of the sequence, the first order is the first term $\left(u_{1}\right)$, the second order is the second term $\left(\mathrm{u}_{2}\right), \ldots$, and the $\mathrm{n}$ order is the $n$ term $\quad\left(u_{n}\right)$ of the integer sequence. Some forms of integer sequences can be said as arithmetic sequences (such as $2,5,8, \ldots, 3 n-1, \ldots$ ), geometric sequence (such as $1,9,27, \ldots, 3^{\mathrm{n}-1}, \ldots$ ), Fibonacci sequence $1,1,2,3,5,8, \ldots)$ and so on. These integer sequences are stated explicitly by using functional formula, each sequence is a function whereas the domain is the set of natural numbers, and the codomain is the set of integers. The statement $u_{n}=$ $2\left(3^{n-1}\right)$ is an explicit functional statement for stating the $\mathrm{n}^{\text {th }}$ term of the sequence, and the statement $\mathrm{u}_{\mathrm{n}}=3 \mathrm{u}_{\mathrm{n}-1}$ is explicitly given for stating the $\mathrm{n}^{\text {th }}$ of the same sequence. The basic concept for implementing the general form is the existence of geometric sequences, the existence of many integer sequences that can be created, and the possible use of recurrence relations to be used for finding the general form of any integer sequences.

In the literature there are many examples of this discourses. One of the integer sequences is a geometric sequence. The general form of a geometric integer sequence is stated explicitly as:

$$
\mathrm{t}, \mathrm{tp}, \mathrm{tp}^{2}, \mathrm{tp}^{3}, \mathrm{tp}^{4}, \ldots, \mathrm{tp}^{\mathrm{n}-1},,,
$$

in which $t$ represents the first term, and $p$ represents the ratio of the geometric sequence. The order of its terms is $\mathrm{u}_{1}=\mathrm{t}, \mathrm{u}_{2}=\mathrm{tp}, \mathrm{u}_{3}=\mathrm{tp}^{2}, \ldots, \mathrm{u}_{\mathrm{n}}=\mathrm{tp}^{\mathrm{n}-1}$, and the sum of the first $\mathrm{n}$ terms is $S_{\mathrm{n}}=\left\{\mathrm{t}\left(\mathrm{p}^{\mathrm{n}-1}-1\right)\right\} /(\mathrm{p}-1)$. One example of a geometric integer sequence is $1,2,4,8$, $16, \ldots, 2^{\mathrm{n}-1}, \ldots$ It is a geometric-integer sequence with $\mathrm{k}=1$ and $\mathrm{p}=2$. The topics of geometric-integer sequence is a part of the mathematics curriculum to be taught at Indonesia schools. It is usually taught implicitly in the elementary school mathematics by exploring number patterns. It was taught explicitly in the middle school mathematics by developing formulas, and it was discussed more deeply in high school mathematics by implementing its application in daily life. However, the discussion about a special sequence created or generated by geometric sequence, such as $1,2,2,4,4,4,4,8,8,8,8,8,8,8,8, \ldots$, is rarely done as a part of the school mathematics curriculum, is rarely discussed in mathematics 
textbooks, and has never brought up as "the implementation" of a geometric-integer sequence.

We can create new sequences from geometric sequences, and it is called integer sequences that are implemented or generated by $\mathrm{t}, \mathrm{tp}, \mathrm{tp}^{2}, \mathrm{tp}^{3}, \mathrm{tp}^{4}, \ldots, \mathrm{tp}^{\mathrm{n}-}$ $1, \ldots$ These new sequences can be written as the list of numbers $\mathrm{t}, \mathrm{tp}, \mathrm{tp}, \mathrm{tp}, \ldots\left[\mathrm{tp}\right.$ times] $\mathrm{tp}^{2}, \mathrm{tp}^{2}, \mathrm{tp}^{2}, \ldots\left[\mathrm{tp}^{2}\right.$ times], .., $\mathrm{tp}^{\mathrm{n}-1}, \mathrm{tp}^{\mathrm{n}-1}, \mathrm{tp}^{\mathrm{n}-1}, \ldots\left[\mathrm{tp}^{\mathrm{n}-1} \mathrm{times}\right], \ldots$, and their terms has a form $\left[\mathrm{u}_{1}=\mathrm{u}_{2}=\mathrm{u}_{3}=\ldots=\mathrm{u}_{\mathrm{t}}=\mathrm{t}\right],\left[\mathrm{u}_{\mathrm{t}+1}\right.$ $\left.=\mathrm{u}_{\mathrm{t}+2}=\mathrm{u}_{\mathrm{t}+3}=\ldots=\mathrm{u}_{\mathrm{t}+\mathrm{p}}=\mathrm{tp}\right],\left[\mathrm{u}_{t+t p+1}=\mathrm{u}_{t+t p+2}=\ldots\right.$ $\left.=\mathrm{u}_{t+t p+t p^{2}}=t^{2}\right], \quad \ldots, \quad\left[\mathrm{u}_{t+t p+t p^{2}+\cdots+1}=\right.$ $\mathrm{u}_{t+t p+t p^{2}+\cdots+2}=\mathrm{u}_{t+t p+t p^{2}+\cdots+3}=\cdots=$ $\mathrm{u}_{\left.t+t p+t p^{2}+\cdots+t p^{n-1}\right]}=\mathrm{tp}^{\mathrm{n}-1}, \ldots$ and we would try to find it rather difficult to find $u_{n}$ for $n$ is big enough. We administer a systematic method as a model for solving the problem. This method was expected to be applicable for simplifying the problem-solving process.

\section{DEVELOPING MODEL}

This is a new model to be used for obtaining a general form of the nth term of specific sequences generated by geometric sequences. The implemented model. It is really very difficult to find out this model because it needs deep observation to relate the geometric sequences and the new generated sequences. The reasoning for obtaining the solution of the problem is creating group elements, in which the number of elements in the group has the rules of geometric sequences. The focus of the model is structuring the first elements of the groups. The way to implement the model will help to make the problem easier to understand, and to give more opportunity to do more open investigation of other integer sequences. The researcher expect that the idea of the created model will be useful and helpful for anyone who is interested in observing and investigating integer sequences generated by integer sequences, especially geometric sequences.

The implemented model is investigating the behavior of the first term of each group of reference sequence. By investigating the facts and inspired by the guessing and testing strategies [1], or guessing and checking method [2], the researcher identifies the pattern of the first term in each group, and the researcher tries to find out a suitable linear recurrence relation with constant coefficient. Then, the recurrence relation is solved by operating the rules in discrete mathematics.. relation:

$$
c_{o} u_{n}+c_{1} u_{n-1}+\ldots+c_{k} a_{n-k}=f(n)
$$

is regarded as a linear recurrence relation (equation) with constant coefficients, and if $\mathrm{f}(\mathrm{n})=0$, it can be known that:

$$
c_{o} u_{n}+c_{1} u_{n-1}+\ldots+c_{k} a_{n-k}=0
$$

and the equation is considered as an homogenous linear equation with constant coefficient.

In solving a homogenous linear recurrence relation with constant coefficients, an approach by substituting $\mathrm{u}_{\mathrm{n}}=\mathrm{r}^{\mathrm{n}}$ into the equationis commonly used, and the following equation is generated:

$$
\mathrm{c}_{\mathrm{o}} \mathrm{r}^{\mathrm{k}}+\mathrm{c}_{1} \mathrm{r}^{\mathrm{k}-1}+\ldots+\mathrm{c}_{\mathrm{k}}=0
$$

This equation is considered as a characteristic of the recurrence relation. The solutions for the characteristic equation are regarded as the characteristic roots of the recurrence relation.

Muhsetyo [4] explained that a homogenous linearrecurrence relation with constant coefficients:

$$
c_{o} u_{n}+c_{1} u_{n-1}+c_{2} u_{n-2}=0
$$

has a characteristic equation:

$$
\mathrm{c}_{\mathrm{o}} \mathrm{r}^{2}+\mathrm{c}_{1} \mathrm{r}+\mathrm{c}_{2}=0
$$

This characteristic equation has one solution $\mathrm{u}_{\mathrm{n}}=\left(\mathrm{t}_{1}+\right.$ $\left.\mathrm{t}_{2} \mathrm{n}\right) \mathrm{r}^{\mathrm{n}}$ or has two different solution $\mathrm{u}_{\mathrm{n}}=\mathrm{t}_{1} r_{1}^{n}+\mathrm{t}_{2} r_{2}^{n}$

The solution of a homogeneous equation is written as $u_{n}^{H}$, the solution of an inhomogeneous equation written as $u_{n}^{P}$, and $u_{n}^{P}$ is regarded as a particular solution.

The solution of an inhomogenous-linear recurrence relation with constant coefficient can be done by finding the homogenous solution $u_{n}^{H}$ and particular solution $u_{n}^{P}$. If the solution is $\mathrm{u}_{\mathrm{n}}$ then:

$$
\mathrm{u}_{\mathrm{n}}=u_{n}^{H}+u_{n}^{P}
$$

\section{DISCUSSION AND IMPLEMENTATION}

To begin with, to make the same perception, a specific sequence that can be obtained from the implementation of a geometric sequence $1,2,4,8,16$, $32, \ldots, 2^{\mathrm{n}-1}, \ldots$ is:

$2^{\mathrm{n}-1} \quad 1,2,2,4,4,4,4,8,8,8,8,8,8,8,8, \ldots, 2^{\mathrm{n}-1}$, $, \ldots, 2^{\mathrm{n}-1}, \ldots$

From the new sequence, it can be written two questions:

(a) find $\mathrm{u}_{100}$, and

(b) find $n$ if $u_{n}=64$

To answer these two questions, the sequence can be stated as a formal sequence: 
$\begin{array}{lllllllllllllllll}1 & 2 & 2 & 4 & 4 & 4 & 4 & 8 & 8 & 8 & 8 & 8 & 8 & 8 & 8 & . & .\end{array}$

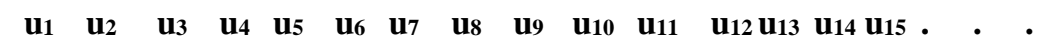

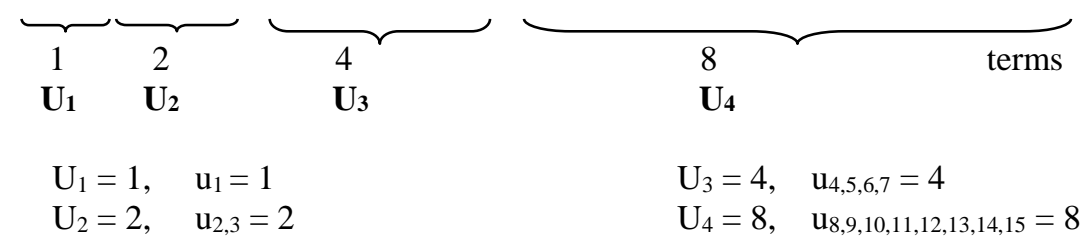

and it can be determined that:

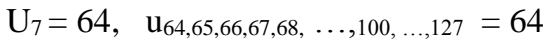

From the last statement can be found that:

(a) $\mathrm{u}_{100}=64$

(b) if $\mathrm{u}_{\mathrm{n}}=64$, then $\mathrm{n}=64,65,66,67, \ldots, 127$

There are some sources for investigating integer sequences [5][6]. There are also many descriptions of integer sequences in OEIS (On-line Encyclopedia of Integer Sequences), and Journal of Integer Sequences (JIS).

The main idea for developing the way to solve the recurrence relation of integer sequences is making a model by creating a new sequence. The model of the new sequence is based on the number of terms of the first (old) sequence, to be the terms of the second (new) sequence. The implementation of the process is focused on the position, or the location of the first order element of the new sequence, it is a sequence of the number of elements in each group. To make the two sequences are different, it can be used $u_{n}$ for stating the nth term of the old sequence, and $U_{n}$ for stating the nth term of the new sequence. a. The Original Sequence

b. The Implemented Sequence

t terms tp terms $\operatorname{tp}^{2}$ terms $\quad \ldots$ $\mathbf{t}, \mathbf{t p}, \mathbf{t p}^{2}, \mathbf{t p}^{3}, \ldots, \mathbf{t p}^{\mathrm{n}-1}, \ldots$

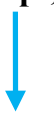

$t, t, t, \ldots, t p, t p, t p, \ldots, t p^{2}, t^{2}, t p^{2}, \ldots$
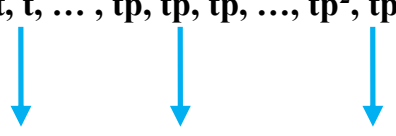

c. Group of Terms

1

2

3

d. Number of Terms

$\mathrm{t}$

tp

$\operatorname{tp}^{2} \quad \cdots$

e. Position of the First Terms

$1+1$

$\mathrm{t}+\mathrm{tp}+1 \quad \ldots$

$\begin{array}{lllllllllllllll}\text { (1) } & \boldsymbol{u}_{1} & \boldsymbol{u}_{2} & \boldsymbol{u}_{3} & \boldsymbol{u}_{t} & \boldsymbol{u}_{t+1} & \boldsymbol{u}_{t+2} & \boldsymbol{u}_{t+3} & \boldsymbol{u}_{t p} & \boldsymbol{u}_{t p+1} & \boldsymbol{u}_{t p+2} & \boldsymbol{u}_{t p+3} & \boldsymbol{u}_{t p^{2}} & \ldots\end{array}$

$\underbrace{\mathrm{t}, \mathrm{t}, \mathrm{t}, \ldots, \mathrm{t}}_{\begin{array}{c}1 \\ \mathbf{U}_{1}\end{array}} \underbrace{\mathrm{tp}, \mathrm{tp}, \quad \mathrm{tp}, \ldots, \mathrm{tp}}_{\begin{array}{c}3 \\ \mathbf{U}_{2}\end{array}}, \underbrace{\mathrm{tp}_{3}^{2}, \mathrm{tp}^{2}, \mathrm{tp}^{2}, \ldots, \mathrm{tp}^{2} \ldots}_{\cdots}$

t terms

(tp) terms

$\left(\mathrm{tp}^{2}\right)$ terms 
To create the integer sequence model for solving the problem, it is used an inhomogeneous recurrence relation with constant coefficient. We need a creative model that the researcher would use can be implemented to be an inhomogeneous recurrence developing a list of element groups in a sequence of numbers as follow:

To identify the functional relation, the list is modified by adding the elements of the first order in each grouP relation with constant coefficients. It is necessary to develop a creative model that can be implemented to be an inhomogeneous recurrence relation with constant coefficients. The steps were begun by

(1) $\begin{array}{lllllllllllllllll}\mathbf{u}_{1} & \mathbf{u}_{2} & \mathbf{u}_{3} & \ldots & \mathbf{u}_{\mathrm{t}} & \mathbf{u}_{\mathrm{t}+1} & \mathbf{u}_{\mathrm{t}+2} & \mathbf{u}_{\mathrm{t}+3} & \ldots & \mathbf{u}_{\mathrm{tp}} & \mathbf{u}_{\mathrm{tp}+1} & \mathbf{u}_{\mathrm{tp}+2} \mathbf{u}_{\mathrm{tp}+3} & \ldots & \mathbf{u}_{\mathrm{t}} p^{2} & \ldots\end{array}$

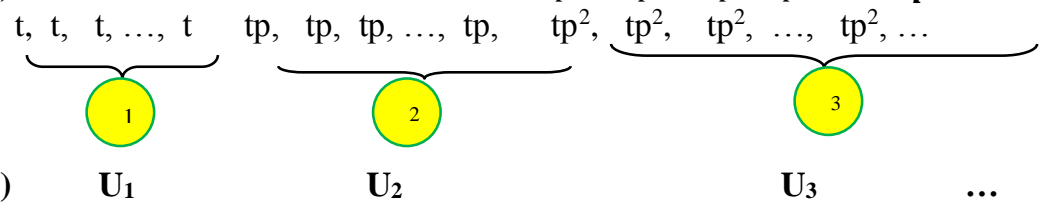

t terms tp terms tp $\mathrm{tp}^{2}$ terms

(3) The created sequence model is related to the first term in each group of terms

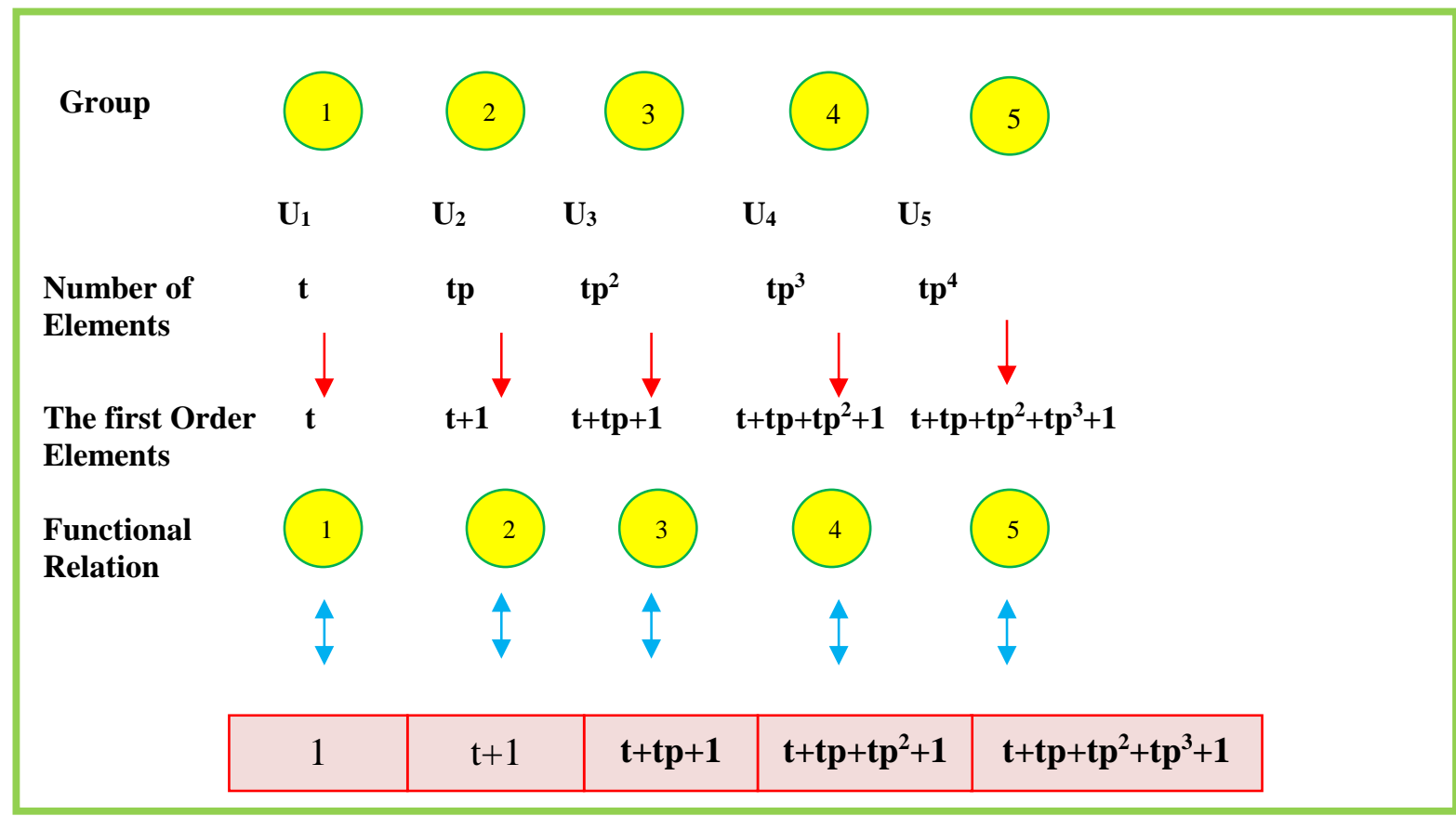

(4) The constructed recurrence relation model

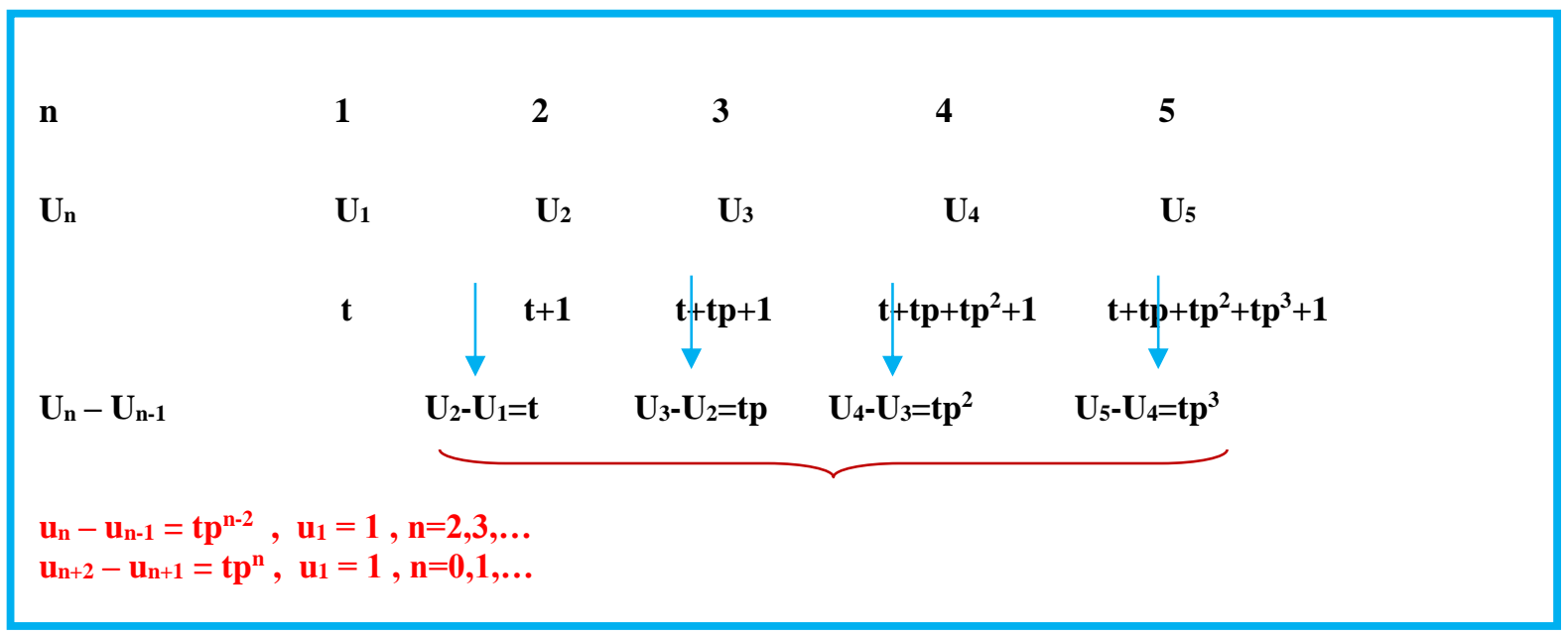




\section{(5) The solution of the constructed linear recurrence}

a. For $\mathrm{t}=2$, and $\mathrm{p}=3$, then the geometric sequence is:

$2,6,18,54, \ldots$

and the implemented sequence generated by the geometric sequence is

$\underbrace{2,2,}_{\begin{array}{c}2 \\ \text { terms }\end{array}}, \underbrace{6,6,6,6,6,6}_{\begin{array}{c}6 \\ \text { terms }\end{array}}, \underbrace{18,18,18,18,18,18,18,18,18,18,18,18,18,18,18,18,18,18}_{\begin{array}{c}18 \\ \text { terms }\end{array}}, 54 \ldots$

terms terms terms

$\mathrm{U}_{\mathrm{n}}=\left(1-\frac{t}{p-1}\right)+\left(\frac{\mathrm{t}}{\mathrm{p}^{2}-\mathrm{p}}\right)\left(\mathrm{p}^{\mathrm{n}}\right)$, then:

$\mathrm{U}_{1}=\left(1-\frac{2}{3-1}\right)+\left(\frac{2}{3^{2}-3}\right)\left(3^{1}\right)=1(2$, the first term in the group 1 , on the first order $)$

$\mathrm{U}_{2}=\left(1-\frac{2}{3-1}\right)+\left(\frac{2}{3^{2}-3}\right)\left(3^{2}\right)=3(6$, the first term in the group 2, on the third order)

$\mathrm{U}_{3}=\left(1-\frac{2}{3-1}\right)+\left(\frac{2}{3^{2}-3}\right)\left(3^{3}\right)=9(18$, the first term in the group 3 , on the ninth order $)$

$\mathrm{U}_{4}=\left(1-\frac{2}{3-1}\right)+\left(\frac{2}{3^{2}-3}\right)\left(3^{4}\right)=27(54$, the first term in the group 4 , on the 27 th order $)$

It is known that $\mathrm{U}_{4}=27$. What is the first term in group 4 ? To answer the problem, it can be seen that the arrangement of the first term of the groups is a geometric sequence with $t=2$ and $p=2$, so from the formula $f_{n}=2.3^{n-1}, f_{4}=2.3^{4-1}=2.3^{3}=54$.

b. For $\mathrm{t}=3$, and $\mathrm{p}=2$, then the geometric sequence is:

$3,6,12,24, \ldots$

and the implemented sequence generated by the geometric sequence is

$3,3,3,6,6,6,6,6,6,12,12,12,12,12,12,12,12,12,12,12,12,24, \ldots$

$\begin{gathered}3 \\ \text { terms }\end{gathered}$
terms $\underbrace{3,3,3,}_{\begin{array}{c}12 \\ \text { terms }\end{array}} \underbrace{6,6,6,6,6,6,} \underbrace{12,12,12,12,12,12}$

$\mathrm{U}_{\mathrm{n}}=\left(1-\frac{t}{p-1}\right)+\left(\frac{\mathrm{t}}{\mathrm{p}^{2}-\mathrm{p}}\right)\left(\mathrm{p}^{\mathrm{n}}\right)$, then:

$\mathrm{U}_{1}=\left(1-\frac{3}{2-1}\right)+\left(\frac{3}{2^{2}-2}\right)\left(2^{1}\right)=1(3$, the first term in the group 1 , on the first order $)$

$\mathrm{U}_{2}=\left(1-\frac{3}{2-1}\right)+\left(\frac{3}{2^{2}-2}\right)\left(2^{2}\right)=4(6$, the first term in the group 2, on the fourth order)

$\mathrm{U}_{3}=\left(1-\frac{3}{2-1}\right)+\left(\frac{3}{2^{2}-2}\right)\left(2^{3}\right)=10(12$, the first term in the group 3 , on the tentth order)

$\mathrm{U}_{4}=\left(1-\frac{3}{2-1}\right)+\left(\frac{3}{2^{2}-2}\right)\left(2^{4}\right)=22(24$, the first term in the group 4 , on the 22nd order)

It is known that $\mathrm{U}_{4}=22$. What is the first term in group 4? To answer the problem, it can be seen that the arrangement of the first term of the groups is a geometric sequence with $t=3$ and $p=2$, so from the formula $f_{n}=3.2^{n-1}, f_{4}=3.2^{4-1}=3.2^{3}=24$.

\section{CONCLUSION AND SUGGESTION}

Investigations of some cases have an important role in deciding the sequence model for solving the recurrence relation generated by a geometric sequence. The model for determining the general term of a sequence is as follow: $\mathrm{t}, \mathrm{t}, \mathrm{t}, \ldots, \mathrm{tp}, \mathrm{tp}, \mathrm{tp}, \ldots, \mathrm{tp}, \mathrm{tp}^{2}, \mathrm{tp}^{2}, \ldots, \mathrm{tp}^{2}, \ldots$

which was developed by creating a new sequence of the first element of a grouping sequence.

From this new sequence, can be identified an inhomogeneous and linear recurrence relation with constant coefficient:

$$
u_{n+2}-u_{n+1}=t^{n}, u_{1}=1, n=0,1, \ldots
$$


By using an undetermined coefficient strategy, the following solution can be obtained:

$$
\mathbf{U}_{\mathrm{n}}=\left(1-\frac{t}{p-1}\right)+\left(\frac{\mathrm{t}}{\mathbf{p}^{2}-\mathbf{p}}\right)\left(\mathbf{p}^{\mathrm{n}}\right)
$$

\section{REFERENCES}

[1] G.I. Musser, W.F. Burger, and B.E. Peterson, Mathematics For Elementary Teachers A Contemporary Approach, Hoboken : John Wiley, 2006.

[2] L.M. Kennedy, S. Tipps, and A. Johnson, Guiding Children's Learning of Mathematics, Belmont Thomson Wadsworth, 2008.

[3] R.P. Grimaldi, Discrete AndCombinatorila Mathematics. New York : Addison-Wesley, 1989

[4] G. Muhsetyo, Matematika Diskrit Tangerang: Universitas Terbuka. 2007.

[5] H.D. Nguyen, Exploring Pattern of Integer Sequences, www.rowanedu/colleges/las/departments/math/faculty/staff/ nguyen/experimental, 2011.

[6] N.J.A. Sloane, The On-Line Encyclopedia of Integer Sequences, Annales Mathematicae at Informaticae, vol. 41, pp. 219-234, 2013. 